\title{
Research on Simulation Technology Application on Higher Education for Deaf/hard-of-hearing Students in China
}

\author{
Dengfeng Yao ${ }^{1,3}$ a , Zhili Liu ${ }^{2, b}$, Junxia Yan ${ }^{4, c}$, Yunfeng Qiu ${ }^{5, d}$ \\ ${ }^{1}$ Beijing Key Laboratory of Information Service Engineering, Beijing Union University, Beijing 100101, China \\ ${ }^{2}$ Special Education College, Beijing Union University, Beijing 100075, China \\ ${ }^{3}$ Lab. of Computational Linguistics, School of Humanities, Tsinghua University, Beijing 100084, China \\ ${ }^{4}$ College of Computer, Wuhan Vocational College of Software and Engineering, Wuhan 430205, China \\ ${ }^{5}$ Foreign Language College, JingChu University of Science and Technology, Jingmen, Hubei 448000, China \\ atjtdengfeng@buu.edu.cn, ${ }^{b}$ tjtzhili@buu.edu.cn, ${ }^{c}$ 826560859.qq.com, dqiuyunfeng200015@gmail.com
}

Keywords: robot simulation; deaf/hard-of-hearing students; educational technology

Abstract: In teaching of computer language, how can we improve their hearing of hearing-impaired students featured with special physiological characteristics and reduce the inconvenience and obstacles caused during teaching knowledge in the most effective way? In this research, we have applied simulation technology and developed the teaching simulation platform for them, and it is practically demonstrated in the hearing-impaired students in higher education and the application of research result that the system works well, with intuitive, efficient, and other characteristics, a strong promotion of the college teaching model changes, the language learning, education teaching and research referred.

\section{Introduction}

With the continuous development of special education, disabled students' education level is constantly improving, so learning computer technology is not the patent of hearing students [1]. Many deaf/hard-of-hearing students are accepted to university and choose Computer Science as their major. Because of their physiological defect, the effect of applying the conventional teaching method into in higher education for deaf/hard-of-hearing students is very poor. In many cases, though we try best, it is still difficult for students to understand fully, which influences the teaching effects directly and restricts the development of special education.

Therefore, we need to practice with the contradictions and problems in the teaching taken into consideration. In the past, as an important part of modern information technology, robot simulation technology is generally applied only for engineering design [2], and there is no experience available in using simulation technology into the high education for deaf/hard-of-hearing students. We account for the characteristics of higher education for the deaf and make a bold attempt to introduce the robot simulation technology to develop simulation teaching platform applied in the deaf/hard-of-hearing students' classroom.

\section{Analysis of teaching elements of the design of simulation platform}

We analyze various contradictions and problems faced in practical teaching, modern simulation technology, their hearing function defects and more sensitive visual perception, trying to introduce the robot simulation technology into the classroom in the teaching, with a set of teaching method suitable for the deaf/hard-of-hearing students worked out. In order to apply this teaching method into the computer programming language courses, we have designed a robot simulation teaching platform that can present computer logic theory through the behavior of the simulation robot whose design principle is shown in figure 1. 


\section{1 design idea:}

\subsubsection{Most basic idea of simulation platform design is using the modern science and technology to help deaf/hard-of-hearing students to achieve "functional compensation".}

Function compensation is using a special teaching method when the handicapped students are educated. For deaf/hard-of-hearing students, function compensation is to use engineering technology to compensate for hearing defects through the visual display or eradicate or make up for the disadvantages caused by learning obstacles in understanding teaching content through animation behavior in order to reduce the learning difficulties caused by barriers. Our goal of design simulation platform is to maximize their advantages of visual acuity and weaken the inconvenience of the auditory defect, so the teaching mode is composite aspects integrated with knowledge and skills, learning and thinking, questions and answers, emotion and investment etc. On this basis, diversified teaching methods combined with the intelligence factors and non-intelligence factors, the process and the result on the equally important position make the teaching activities conducted on the basis of knowledge that can be accepted and consistent with the level of cognitive development[3].

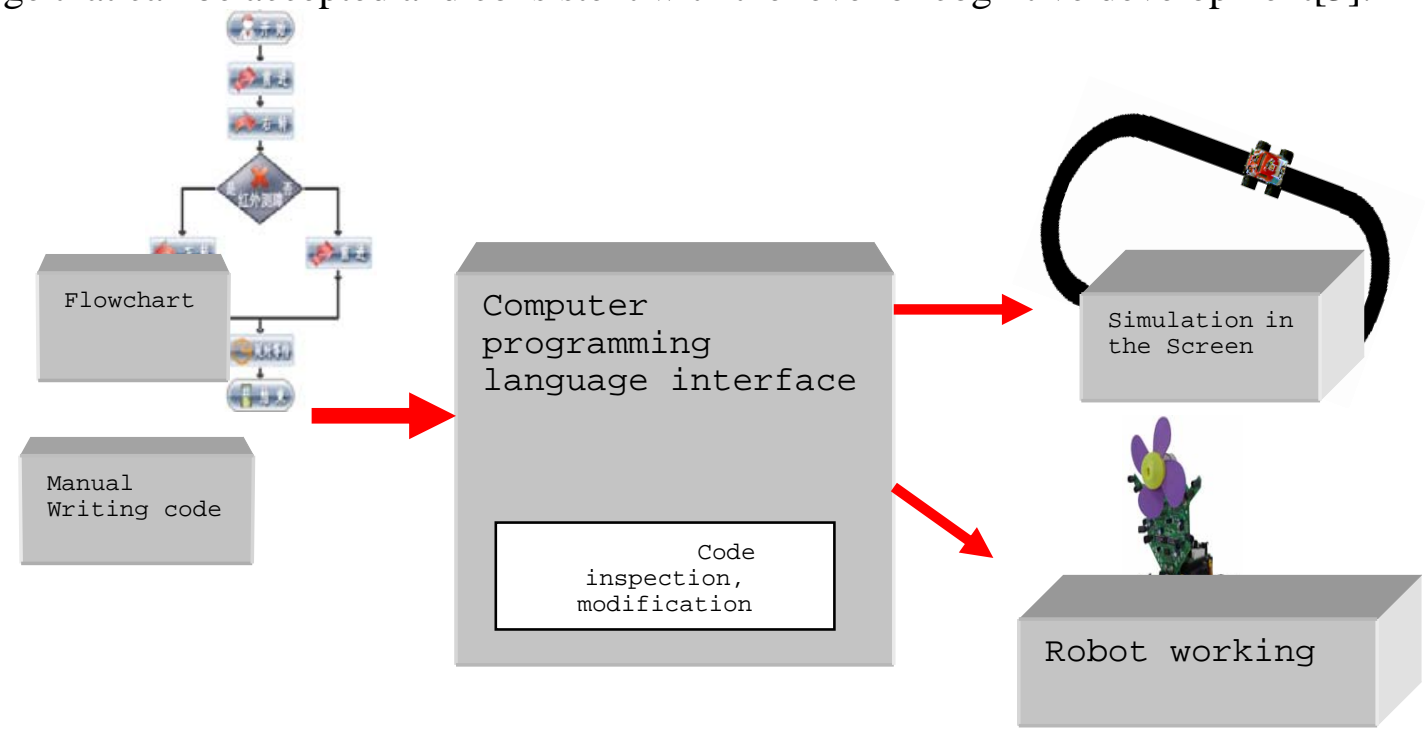

Figure1 detailed schematic design

\subsubsection{To adopt Engineering project design}

We have combined difficult points encountered by deaf/hard-of-hearing students during learning the computer programming language and teaching knowledge points demonstrated by the simulation methods thought of as new teaching methods. For example: to interpret the relationship between flow chart and the programming language instruction, we can put aside the technical terms that are not easy to understand and design a new teaching mode where [4] flow chart corresponds to programming language instruction in the engineering design project [5], and teaching methods can make deaf/hard-of-hearing students discover written instructions explicitly corresponding to executed behavior of the simulation robot. The platform software applies the project practice training to cultivate the spirit of cooperation through every teaching procedure such as learning, search, collection, communication, design and running. (See Figure 2).

\subsection{Analysis of the teaching task and learning objects}

When making the teaching task, we need to design the established goals that can be reached successfully through a series of efforts according to the syllabus requirements and the actual situation of the deaf. In order to achieve the established objectives, teachers must analyze many possible factors including students' learning enthusiasm, initiative of participation, as well as their mental processes, which stops students from realizing the goal. For example, in the past, it is found that they are more likely to give up these knowledge points when deaf/hard-of-hearing students learn computer programming language and are afraid of difficulties severely, although these knowledge points seem to be extremely simple for hearing students.

In the design of teaching simulation platform, we make careful analysis and find that the key factors of achieving the teaching goal is not only the teaching content, way and method, but also 
physical and psychological feature and cognitive ability of teaching object. The relation between teaching and learning is kind of interactive. Therefore, in designing the teaching goal we must consider the situation above mentioned. We need using the appropriate methods and take the "teaching" and "learning" side’s actual situation, into account to complete really the teaching task, letting students fully understand the content.

\section{Engineering Project}
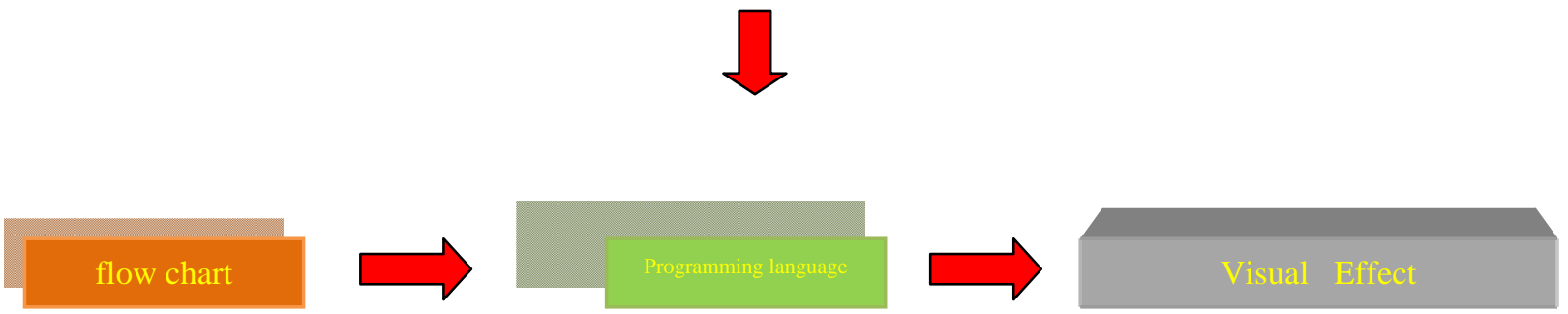

Figure2 teaching design scheme

\subsection{Analysis of the teaching process}

The design of teaching process is to design a series of steps that can be operated. Before teaching, we need to analyze their knowledge background and the teaching means needed to complete in order to meet the urgent requirements of realizing teaching goals. Teaching is to "teach" the deaf "to learn", which is not simply to put the textbook knowledge to deaf students, but teach them the way and approach to learning knowledge.

1.3.1 As for teaching content, we need to determine how the gap between the knowledge and skills required is and their original knowledge base bridges the gap. We hope to construct an appropriate knowledge bridge. Namely, starting from basic knowledge in which they are lack to their new knowledge, we set up a suitable knowledge transition bridge so as to pass through the bridge as soon as possible for deaf/hard-of-hearing students, gain the ability to learn new knowledge, and achieve the teaching goal.

1.3.2teaching method [6] was determined according to the teaching content. Assisted by the simulation teaching platform, we can guide students to set up the basic concept of engineering design so that the deaf/hard-of-hearing students can express their idea about project design by means of computer simulation, and use this method to complete the custom project practice.

1.3.3 To design the teaching process, we select the breakthrough point, according to the physiological characteristics and knowledge level of deaf/hard-of-hearing students. The teachers should "activate" book knowledge whose main carrier is symbols, and work out a personalized reading and interpretation [7]. As for textbooks, it is necessarily important for teachers to make efforts to make abstract symbol theory easily and concisely understood. In addition, teachers are supposed to direct the deaf/hard-of-hearing students in learning to choose and use the tasks properly, for example, deaf/hard-of-hearing students should learn to divide content into a series of partially easy processing tasks to obtain a more relaxed learning method. Only through feeling that this research is so useful and funny can they construct the dynamic knowledge system in the experiences process, learn how to learn and enjoy learning.

\subsection{Treatment of several relations in platform design}

\subsubsection{The relationship between teaching goal and process}

In general computer language teaching starts roughly from the command structure and timely analysis. Although this teaching mode is featured with standard significance, it builds a barrier for deaf/hard-of-hearing students to understand. Simulation teaching platform de-emphasizes teaching theme in teaching according to the differences between deaf and hearing students in stead of running the effort of program implementation by platform software in a flashbacks way to interpret language instructions used in the programming to inspire and guide them in learning programming language. Then, we can help deaf/hard-of-hearing students through various forms. 


\subsubsection{Relationship with the combination of decomposition}

"Teaching platform" can be used to complete a task (project) by organizing teaching process in order to achieve the teaching purpose from the decomposition to the combination. A complete program can be divided into a number of subroutines in which "teaching platform" contains a series of tasks such as programming, simulation robot, simulation field etc. that the deaf can independently operate or collaborate.

\section{Conclusion and future work}

Deaf teaching simulation platform is designed to solve the practical problems in the teaching process for deaf/hard-of-hearing students, at the same time; it can also be applied for hearing students, especially for those whose basic knowledge is weak with no abstract thinking ability. Our platform can make them easily learn computer programming language because it will demonstrate those abstract terms and program structure that are difficult for them by the animated simulation so as to make it easy to understand.

Introducing simulation technology into teaching computer programming language for deaf/hard-of-hearing students is a new topic which narrows the gaps in the field of higher special education. Implement of this advanced teaching ideas and methods also improve the teaching level of deaf/hard-of-hearing students and establish a foundation for other related computer application courses.

Secondly, the laboratory condition remains to be perfected. At present, CAI courseware resource available for deaf/hard-of-hearing students is relatively insufficient, and the development of the teaching platform for oriented object programming language is toned to be researched and developed. For example, we need to study how to apply this platform in teaching oriented-object programming language by the popular $\mathrm{VC}++$ software.

Finally, The shortcomings of the simulation platform is mainly manifested in that the object-oriented idea and application development is not fully demonstrated by simulation technology and how all kinds of logical relations in the software development are transformed into virtual action of simulation robot remains for further research and development so as to cultivate deaf/hard-of-hearing students’ abstract knowledge ability.

\section{Acknowledgements}

This paper has been supported by the National Natural Science Foundation of China (61171114), Beijing Higher Education Young Elite Teacher Project (YETP1753), the Project of Construction of Innovative Teams and Teacher Career Development for Universities and Colleges Under Beijing Municipality (IDHT20130513), the Importation and Development of High-caliber Talents Project of Beijing Municipal Institutions (IDHT201304073), and “New Start” Academic Research Projects of Beijing Union University (2K201213).

\section{References}

[1] Lin Jin, Liu Changan, Wu kehe, etc. Design and implement the Robot simulation. Computer Application and Research, 2003, (10). 138 140.

[2] Higher education for disabled, http://www.cdpf.org.cn/jiaoyu/jy0004.htm

[3] Patricia A. Mudgett-DeCaro, James J. DeCaro, Postsecondary Education for Deaf People in China: Issues, Roles, Responsibilities and Recommendations, http://www.pen.ntid.rit.edu/pdf/chinarpt06.pdf

[4] National Standard Of the People's Republic of China - information processing flow chart graphic symbols, the national standard GB1526-89

[5] Tajon. Messom C1 Deterimining offensive and defensive procession in multiagent team based competitive games. Korea. Proc. Of Microsoft, 1997.71 75

[6] Xuesen Qian. Thinking Science. Shanghai: Shanghai People's Publishing House, 1986.137 141

[7] Ministry of Education. Higher Education. Higher Education Press,2003 